\title{
Etiología y caracterización epidemiológica del síndrome febril no palúdico en tres municipios del Urabá antioqueño, Colombia
}

\author{
Esteban Arroyave ${ }^{1}$, Andrés Felipe Londoño', Juan Carlos Quintero', Piedad Agudelo-Flórez², \\ Margarita Arboleda ${ }^{2}$, Francisco J. Díaz ${ }^{3}$, Juan D. Rodas ${ }^{1}$ \\ 1 Grupo Centauro, Universidad de Antioquia, Medellín, Colombia \\ 2 Instituto Colombiano de Medicina Tropical, Universidad CES, Medellín, Colombia \\ ${ }^{3}$ Grupo de Inmunovirología, Universidad de Antioquia, Medellín, Colombia \\ Lugar donde se realizó la investigación: \\ Grupo Centauro de la Universidad de Antioquia e Instituto Colombiano de Medicina Tropical de la Universidad \\ CES, Medellín, Colombia.
}

Introducción. La región de Urabá es endémica para varias enfermedades febriles agudas de origen infeccioso. Sin embargo, solo los pacientes con malaria pueden acceder a un diagnóstico oportuno y rápido, motivo por el cual muchos síndromes febriles no palúdicos quedan sin diagnóstico etiológico claro.

Objetivo. Establecer la etiología, describir las manifestaciones clínicas y explorar algunos posibles factores de riesgo relacionados con los síndromes febriles agudos no palúdicos en pacientes procedentes de los municipios de Necoclí, Turbo y Apartadó.

Materiales y métodos. Se tomaron muestras de suero en fase aguda y de convalecencia de 220 pacientes febriles negativos para malaria, provenientes de zonas rurales y urbanas de Necoclí, Turbo y Apartadó en los años 2007 y 2008. Se practicaron pruebas para diagnóstico de dengue (detección de anticuerpos IgM por ELISA), leptospirosis (detección de anticuerpos IgM e IgG por IFI), rickettsiosis (detección de anticuerpos IgG por IFI), hantavirus y arenavirus (detección de anticuerpos IgG por ELISA).

Resultados. Se encontraron frecuencias de dengue, leptospirosis, rickettsiosis y arenavirus de 37,3 \%, $14,1 \%, 2,7 \%$ y $0,5 \%$, respectivamente. Se presentaron 12 casos de coinfección de leptospirosisdengue y uno de leptospirosis-rickettsiosis-dengue. El sexo masculino y la humedad relativa media, fueron factores de riesgo para dengue. El inicio de signos clínicos en febrero de 2008, se asoció tanto con la infección por dengue como por leptospirosis.

Conclusión. Se reafirma la importancia del virus del dengue, Rickettsia spp. y Leptospira spp., como agentes causantes del síndrome febril en la región del Urabá.

Palabras clave: zoonosis, vigilancia epidemiológica, pruebas serológicas, síndrome febril, Colombia. doi: http://dx.doi.org/10.7705/biomedica.v33i0.734

Etiology and epidemiological characterization of non-malarial febrile syndrome in three municipalities of Urabá (Antioquia), Colombia

Introduction: Urabá, a region on the northern coast of Colombia, is endemic to several acute febrile illnesses of infectious origin; however, only patients with malaria may have access to quick and effective diagnosis. For this reason, many non-malarial febrile patients go without a clear etiologic diagnosis. Aim: To establish the etiology and clinical signs of acute febrile non-malaria syndromes and explore some of the likely risk factors in patients originating in the municipalities of Necocli, Turbo and Apartado who exhibit these symptoms.

Materials and methods: We obtained acute and convalescent sera from 220 non-malarial febrile patients from the rural and urban zones of Necocli, Turbo and Apartado during 2007 and 2008. Serologic

\footnotetext{
Contribución de los autores:

Esteban Arroyave: trabajo de laboratorio, redacción del manuscrito.

Andrés Felipe Londoño: recolección de las muestras, pruebas serológicas virales, discusión y redacción del manuscrito. Juan Carlos Quintero: análisis estadístico y pruebas de inmunofluorescencia para Rickettsia.

Piedad Agudelo-Flórez: diseño del estudio, análisis de la información y revisión del manuscrito.

Margarita Arboleda: análisis de la información, contribución con la discusión y revisión del manuscrito.

Francisco J. Díaz: diseño del estudio, análisis de la información y revisión del manuscrito.

Juan D. Rodas: planeación del trabajo, obtención de recursos para el estudio, análisis de la información y corrección final del manuscrito.
} 
tests for dengue (IgM by ELISA), leptospirosis (IgM and IgG by IFA), rickettsiosis (IgG by IFI), hanta and arenavirus (IgG by ELISA) were performed.

Results: We found that the frequency of infection for dengue, leptospirosis, rickettsiosis and arenavirus, was $37.3 \% ; 14.1 \% ; 2.7 \%$ and $0.5 \%$, respectively. There were 12 co-infection cases of leptospirosis-dengue and one of leptospirosis-rickettsiosis-dengue. Male gender and relative humidity were considered risk factors for dengue, and the beginning of clinical signs in February of 2008 was associated with the infection of dengue and leptospirosis.

Conclusion: This study confirms previous records that underline the importance of Rickettsia spp, dengue virus and Leptospira spp as causal agents of febrile syndrome in this region of Colombia.

Key words: zoonoses, epidemiological surveillance, serological tests, febrile syndrome, Colombia. doi: http://dx.doi.org/10.7705/biomedica.v33i0.734

En 1998, la Organización Mundial de la Salud (OMS) propuso modificar el sistema de vigilancia internacional de enfermedades de reporte obligatorio, bajo el concepto de vigilancia de síndromes, en el cual se reconocen seis diferentes entidades: síndrome febril, síndrome febril ictérico agudo, síndrome febril con manifestaciones hemorrágicas, síndrome febril respiratorio agudo, síndrome febril con manifestaciones neurológicas y síndrome febril con erupción dérmica $(1,2)$. El objetivo de esta medida es hacer más sensible la vigilancia de los sistemas de salud y permitir detectar rápidamente los brotes o enfermedades que pongan en riesgo la salud de la población (1).

El síndrome febril agudo es definido como el estado mórbido con inicio repentino de fiebre, de menos de siete días de evolución, en pacientes entre 5 y 65 años, en el cual no se hayan identificado signos o síntomas relacionados con un foco infeccioso (2). Debido a que estos signos se presentan en forma frecuente y se asocian con sintomatología inespecífica, la Organización Mundial de la Salud (OMS) mediante el Reglamento Sanitario Internacional determinó que en todas las situaciones en las cuales se presente alguno de los síndromes mencionados, estos deben ser notificados obligatoriamente, sin esperar a que el agente causal sea identificado, para así poder lograr una detección precoz de los casos a fin de evitar las epidemias locales y la propagación internacional de la enfermedad (1). No obstante, se deben hacer esfuerzos para identificar los agentes causantes del síndrome febril, y así lograr mejorar las medidas de prevención y control de estas enfermedades.

\footnotetext{
Correspondencia:

Juan D. Rodas, Centro de Investigaciones Agrarias, Facultad de Ciencias Agrarias, Carrera 75 N 65-87, Ciudadela de Robledo, Universidad de Antioquia, Medellín, Colombia

Teléfono: (574) 219 9119; fax: 2199111

juandavid.rodas@gmail.com

Recibido: 10/05/12; aceptado:07/03/13
}

Entre las enfermedades infecciosas que se manifiestan como síndromes febriles agudos en las Américas, se encuentran los siguientes: malaria, bartonelosis, dengue, fiebres de Mayaro y Oropuche, hantavirosis, encefalitis equina venezolana, arbovirosis del grupo C, influenza, rickettsiosis, leptospirosis, brucelosis, salmonelosis, peste bubónica, ehrlichiosis, fiebre $Q$, hepatitis A, B y C, sarampión, rubéola y enfermedad de Lyme, entre otras (2). Sin embargo, la reconocida situación epidemiológica de Colombia con respecto a la diseminación de vectores como Aedes aegypti y Anopheles spp. $(3,4)$, ha llevado a que se reconozcan sólo algunas enfermedades como prioritarias, existiendo un mayor énfasis en dengue, fiebre amarilla y malaria.

La malaria, o paludismo, es la principal causa de síndrome febril agudo que se diagnostica en la región del Urabá antioqueño, localizada al noroeste del departamento de Antioquia, en el golfo de Urabá. En el 2010, se notificaron a la Dirección Seccional de Salud de Antioquia 7.207 casos de malaria procedentes de la región de Urabá. Debido a que la malaria comparte la misma presentación clínica inicial con otros síndromes febriles de origen infeccioso, como los descritos, es posible que algunos agentes etiológicos diferentes no estén siendo diagnosticados, desconociéndose su impacto sobre la salud en esta población (5).

En la región de Urabá se han llevado a cabo varios trabajos epidemiológicos, buscando establecer la seroprevalencia de agentes causantes de síndrome febril. En los estudios de leptospirosis se registran seroprevalencias que van desde 7,5 $\%$ hasta $35,6 \%$ (5-7) y, de dengue, entre $50 \%$ y $77,7 \%$ (8). En el 2010, la Dirección Seccional de Salud de Antioquia reportó, para la zona de Urabá, 250 casos de dengue clásico, 14 casos de dengue hemorrágico y 85 casos de leptospirosis (9). Asimismo, se ha venido documentando la circulación de otros agentes poco comunes en la 
zona, como las rickettsias, con dos brotes en los municipios de Necoclí y Turbo en los años 2006 y 2008, respectivamente, y otro en Los Córdobas (Córdoba) en límites con el departamento de Antioquia en el $2007(10,11)$. Además, también se han encontrado pruebas serológicas y genéticas de la circulación de hantavirus en roedores de la zona de Urabá (12).

Con el presente estudio se pretende contribuir al conocimiento de la causa y la presentación clínica de síndromes febriles agudos no palúdicos, y determinar algunos posibles factores de riesgo asociados, en pacientes febriles procedentes de los municipios de Necoclí, Turbo y Apartadó.

\section{Materiales y métodos}

\section{Obtención de la muestra}

Este es un estudio de tipo descriptivo, longitudinal y retrospectivo, en el que la selección de los participantes se hizo por conveniencia, incluyendo los pacientes que ingresaban a los centros de salud de cada municipio con historia de síndrome febril no palúdico es decir, con gota gruesa negativa como criterio de inclusión. Como caso se consideró a todo paciente con inicio brusco de fiebre (temperatura axilar mayor o igual a $38^{\circ} \mathrm{C}$ ) de menos de siete días de evolución, que tuviese entre 5 y 65 años de edad, con manifestaciones hemorrágicas o sin ellas. El estudio se llevó a cabo de septiembre de 2007 a agosto de 2008. Durante este período, se incorporaron 220 pacientes aptos para el estudio, procedentes tanto de área rural como urbana de los municipios de Necoclí, Turbo y Apartadó.

A todos los que participaron en el estudio se les tomaron dos muestras de sangre para la determinación de anticuerpos en suero. Las muestras para serología se tomaron durante los primeros cinco días a partir de ingreso al centro de salud (fase aguda) y entre 15 y 20 días después de la toma de la primera muestra (fase de convalecencia). Estas muestras fueron almacenadas $\mathrm{a}-20^{\circ} \mathrm{C}$.

\section{Pruebas serológicas}

\section{Leptospira}

Para la determinación de serología positiva para Leptospira spp., se utilizó inicialmente una inmunofluorescencia indirecta (IFI) para la detección de IgM e IgG como prueba tamiz de género, utilizando el antígeno L. biflexa del serogrupo Semaranga y del serovar Patoc, como representativo de todas las serovariedades registradas. Se utilizó una dilución de los sueros de 1:50. A los sueros positivos se les hizo la prueba de microaglutinación (MAT) para determinar el serogrupo responsable de la respuesta de anticuerpos. Los antígenos para esta prueba se obtuvieron del cepario disponible en el Laboratorio de Microbiología del Instituto Colombiano de Medicina Tropical, Universidad CES.

Los sueros se probaron con 10 serogrupos de Leptospira: Semaranga, Ballum, Icterohaemorrhagiae, Grippotyphosa, Pomona, Canicola, Bratislava, Shermani, Pyrogenes y Tarassovi. Esta prueba se practicó según las recomendaciones de la OMS y de acuerdo con lo propuesto por Agudelo Flórez, et al,. $(13,14)$. Para la confirmación serológica de los casos, se consideraron positivos los sueros que en la IFI presentaron un título superior a 1:40 para la IgM o un cambio de dos diluciones en el título de lgG entre la muestra de fase de convalecencia y la de la fase aguda (alza cuádruple). Según la prueba de microaglutinación, se consideraron positivos los sueros que presentaron títulos con una diferencia de dos diluciones dobles entre la muestra de la fase de convalecencia y la de la fase aguda, con cualquiera de los serogrupos patógenos probados.

\section{Rickettsia}

Para el diagnóstico de infección por Rickettsia spp., se determinó la presencia de anticuerpos IgG contra agentes del grupo de las fiebres manchadas mediante IFI, siguiendo el método citado por Horta, et al,. (15) y utilizando placas con antígenos de Rickettsia rickettsii, donadas por Marilyn Hidalgo, del Instituto Nacional de Salud.

A los pozuelos de dichas placas se les adicionó $10 \mu \mathrm{l}$ de la muestra de suero la muestra en una dilución de 1:64. Se incluyó un suero como control negativo y otro como control positivo por cada placa. Posteriormente, las placas se incubaron en una cámara húmeda durante 30 minutos a una temperatura de $37 \pm 0,5{ }^{\circ} \mathrm{C}$ y se lavaron dos veces con solución tampón de lavado después de la incubación. A continuación, se adicionó una gota $(10-20 \mu \mathrm{l})$ de conjugado (IgG anti-humano conjugado con FITC) por pocillo y se incubó nuevamente la placa en cámara húmeda durante 30 minutos a $37 \pm 0,5{ }^{\circ} \mathrm{C}$. Acto seguido, se lavó dos veces con solución tampón de lavado con azul de Evans, leyéndose posteriormente en un microscopio de fluorescencia. Se consideraron como positivos aquellos sueros que reaccionaron en una dilución $\geq 1: 64$ y, como casos confirmados, aquellos que presentaron una diferencia cuádruple de título entre el suero de la fase de convalecencia y el de la fase aguda. 


\section{Hantavirus y arenavirus}

Para el diagnóstico de infección por hantavirus y arenavirus, se practicó una ELISA (Enzyme-Linked ImmunoSorbent Assay) para detectar IgG. Esta técnica consiste en sembrar antígeno en la fase sólida de un microplato, con un lisado de células Vero infectadas con el virus Maciel para hantavirus o con la cepa XJC13 del virus Junín para arenavirus, dejándose toda la noche a $4{ }^{\circ} \mathrm{C}$. Después del lavado, se adicionan el suero problema diluido a 1:400 y los controles, y se lleva a incubación durante una hora a $37^{\circ} \mathrm{C}$. Se lava nuevamente y se aplica el conjugado marcado con peroxidasa anti-lgG humana, incubándose igual que en el paso anterior. Se lava nuevamente y se revela adicionando un sustrato (TMB), el cual se deja 20 minutos a temperatura ambiente y se para la reacción con ácido sulfúrico $1 \mathrm{M}$. Por último, el microplato se lee en un espectrofotómetro con dos filtros, con longitud de onda de 450 y $630 \mathrm{~nm}$, y los sueros que presenten una densidad óptica por encima de 0,2 se consideran reactivos. Los pacientes que presentaron un cambio de título de dos diluciones dobles (alza cuádruple) entre la muestra de fase de convalecencia y la de la fase aguda, se consideraron casos confirmados.

\section{Dengue}

La ELISA para detectar anticuerpos IgM contra el virus del dengue se practicó con el estuche de ELISA de IgM de captura, producido por la casa comercial Focus Diagnostics, siguiendo las instrucciones del inserto adjunto. Los sueros de los pacientes y los controles se diluyeron a 1:100 en el reactivo de dilución y se adicionaron $100 \mu \mathrm{l}$ a cada pozo, que contenía un anticuerpo específico anti-lgM humano. Se incubó durante una hora a temperatura ambiente y se lavó. Después, se adicionaron $100 \mu \mathrm{l}$ de los antígenos de dengue en cada pozo, se incubó como en el paso anterior, se lavó nuevamente y se aplicaron $100 \mu \mathrm{l}$ del conjugado (anti-flavivirus producido en ratón). Se dejó incubando 30 minutos a temperatura ambiente, se lavó y se agregó el sustrato, se incubó 10 minutos y se paró la reacción. Por último, se leyó en un espectrofotómetro a una longitud de onda de $450 \mathrm{~nm}$. Los sueros positivos fueron todos aquellos que presentaron una densidad óptica tres veces por encima de los puntos de corte de la prueba. Se consideró caso confirmado a aquellos que presentaban IgM positiva en la fase de convalecencia y negativa en la fase aguda.

\section{Análisis estadístico}

El análisis descriptivo se hizo a partir de una encuesta en la cual se registraron los signos y síntomas de los pacientes con síndrome febril. Se utilizaron cuadros de frecuencia para mostrar la distribución de todas las variables consultadas (sociodemográficas, saneamiento básico, convivencia con animales y exposición a ectoparásitos).

Para los factores de riesgo y de protección, se desarrolló un análisis de regresión logística para cada una de las enfermedades que presentaron mayor frecuencia (dengue, leptospirosis y rickettsiosis). La variable dependiente fue la enfermedad y las variables independientes fueron las sociodemográficas, signos clínicos, variables medioambientales y variables espaciales. En el modelo final multivariado, se tuvieron en cuenta las variables significativas $(p<0,05)$ y se validaron los supuestos de multicolinearidad mediante una matriz de correlación de Spearman. Los análisis estadísticos se hicieron en el paquete estadístico SPSS ${ }^{\text {TM }}$, versión 18.

A todos los pacientes que ingresaron en el estudio se les solicitó el consentimiento informado por escrito. Además, los procedimientos llevados a cabo dentro de esta investigación fueron avalados por el Comité de Bioética de la Universidad de Antioquia.

\section{Resultados}

\section{Características sociodemográficas}

De acuerdo con los criterios definidos en el presente estudio, fue posible identificar 220 pacientes febriles entre la población que demandaba atención en los establecimientos de salud de cada municipio. La distribución por procedencia fue como sigue: Turbo con 80 pacientes (36,6\%), Apartadó con 77 (35\%) y Necoclí con 43 (19,6\%). Además, se presentaron 20 pacientes $(9,1 \%)$ que pertenecían a otros municipios del Urabá (Unguía, Murindó, Carepa y Chigorodó), los cuales fueron atendidos en los centros de salud donde se hizo el estudio. Estos últimos fueron excluidos únicamente para el análisis de las características sociodemográficas, debido a que los datos eran muy pocos para poder hacer asociaciones sobre el comportamiento de los agentes etiológicos en una población puntual.

En el cuadro 1 se presentan los resultados obtenidos en relación con las características sociodemográficas de los pacientes febriles identificados en los tres municipios en estudio.

En esta muestra se encontró un predominio significativo del sexo masculino (72,7\%) únicamente en el municipio de Apartadó. En lo que se refiere a la edad, se observó una mayor frecuencia en los 
Cuadro 1. Características sociodemográficas de los pacientes febriles en los establecimientos de salud de tres municipios del Urabá antioqueño, 2007-2008

\begin{tabular}{|c|c|c|c|c|c|c|}
\hline \multirow{3}{*}{ Variable } & \multicolumn{6}{|c|}{$\begin{array}{l}\text { Municipios de atención hospitalaria y } \\
\text { procedencia de los pacientes con } \\
\text { síndrome febril }\end{array}$} \\
\hline & \multicolumn{2}{|c|}{ Apartadó } & \multicolumn{2}{|c|}{ Turbo } & \multicolumn{2}{|c|}{ Necoclí } \\
\hline & $n$ & (\%) & $\mathrm{n}$ & $(\%)$ & $\mathbf{n}$ & $(\%)$ \\
\hline Pacientes & 77 & $(35,0)$ & 80 & $(36,4)$ & 43 & $(19,5)$ \\
\hline \multicolumn{7}{|l|}{ Sexo } \\
\hline Masculino & 56 & $(72,7)$ & 38 & $(47,5)$ & 24 & $(55,8)$ \\
\hline Femenino & 21 & $(27,3)$ & 42 & $(52,5)$ & 19 & $(44,2)$ \\
\hline \multicolumn{7}{|l|}{ Edad (años) } \\
\hline$<10$ & 17 & $(22,1)$ & 8 & $(10,0)$ & 9 & $(20,9)$ \\
\hline $11-15$ & 3 & $(3,9)$ & 5 & $(6,2)$ & 4 & $(9,3)$ \\
\hline $16-20$ & 10 & $(13,0)$ & 12 & $(15,0)$ & 5 & $(11,6)$ \\
\hline $21-25$ & 11 & $(14,3)$ & 4 & $(5,0)$ & 2 & $(4,6)$ \\
\hline $26-30$ & 8 & $(10,4)$ & 9 & $(11,2)$ & 4 & $(9,3)$ \\
\hline $30-40$ & 13 & $(16,9)$ & 21 & $(26,2)$ & 3 & $(7,0)$ \\
\hline$>40$ & 15 & $(19,5)$ & 21 & $(26,2)$ & 16 & $(37,2)$ \\
\hline \multicolumn{7}{|l|}{ Zona } \\
\hline Rural & 12 & $(15,6)$ & 34 & $(42,5)$ & 29 & $(67,4)$ \\
\hline Urbana & 65 & $(84,4)$ & 46 & $(57,5)$ & 14 & $(32,6)$ \\
\hline
\end{tabular}

*20 de los pacientes muestreados atendidos en estos hospitales, procedían de los municipios de Unguía, Murindó, Carepa y Chigorodó, y debido a su baja frecuencia, fueron excluidos del análisis sociodemográfico.

grupos etarios de menores de 10 y de mayores de 40 años, en Apartadó y en Necoclí (cuadro 1). En Turbo, los grupos etarios más frecuentes fueron los de 30 a 40 años y los mayores de 40 años. Asimismo, en Turbo se observó un aumento significativo en los individuos entre 26 y 40 años (37,5\%).

Cuando se analiza la procedencia de los pacientes con síndrome febril, se destaca el hecho de que el $84,4 \%$ de los casos procedía de la zona urbana en Apartadó y, el 67,4 \% de los casos, de la zona rural en Necoclí, y hubo una distribución similar para ambas zonas en el municipio de Turbo.

\section{Diagnóstico etiológico}

Del total de casos con síndrome febril que ingresaron al estudio (220), en $37,3 \%$ (82) se diagnosticó dengue; en 14,1\% (31), leptospirosis; en 2,7\% (6), rickettsiosis; en 0,5\% (1), infección por arenavirus; en ninguno, hantavirus y, en el 57,3\% restante (115), no fue posible determinar la causa. En algunos pacientes se evidenció reacción sin cambio en el título de anticuerpos: para rickettsias, $46(20,1 \%)$, para leptospira, 5 (2,3\%), para hantavirus, $2(0,9 \%)$ y para arenavirus, $1(0,5 \%)$; estos valores determinan únicamente exposiciones previas al agente.
Vale la pena aclarar que, entre los individuos infectados, se presentaron 15 coinfecciones distribuidas así: en 5,4 \% (12), leptospirosisdengue; en $0,9 \%$ (2), rickettsiosis-dengue, y en $0,4 \%$ (1), leptopirosis-rickettsiosis-dengue.

De los 31 pacientes positivos por IFI para Leptospira, 10 fueron reactivos por microaglutinación, 9 de los cuales tuvieron títulos significativos para el serogrupo Tarassovi y uno para el serogrupo Semaranga.

En el cuadro 2 se presenta el total de casos reactivos en cada municipio. Se destaca, entre otros datos, la presentación de dengue en los tres municipios de estudio, la ausencia de leptospirosis en Necoclí y los títulos significativos para Rickettsia spp., aunque sin seroconversión, en 17, 15 y 11 pacientes de los municipios de Necoclí, Turbo y Apartadó, respectivamente.

La distribución por sexo, edad y procedencia según la enfermedad, se muestra en el cuadro 3. El sexo masculino predominó en todos los diagnósticos. Con respecto a la edad, el dengue fue más frecuente en la población mayor de 30 años, a diferencia de Leptospira y Rickettsia, que no mostraron diferencias según el grupo etario. Asimismo, se observa una mayor frecuencia de casos de dengue y leptospirosis en la zona urbana $(62,2 \% ; p=0,0030$ y $71 \% ; p=0,0023$, respectivamente) y una mayor frecuencia de rickettsiosis en la zona rural $(66,7 \%$; debido a que el número de casos de rickettsiosis es solo de seis, no es significativo definir un valor $p$ ).

Se presentó concomitancia principalmente entre leptospira y dengue (12 casos), distribuidos en los municipios de Apartadó (4) y Turbo (8), entre Rickettsia y dengue, en dos casos de Turbo, y en un solo individuo de Apartadó se detectaron simultáneamente los tres agentes, Leptospira, Rickettsia y virus del dengue.

\section{Características clínicas de los pacientes}

Las manifestaciones clínicas de los tres diagnósticos más frecuentes se presentan en el cuadro 4, donde se destacan la fiebre, la cefalea y las mialgias como los síntomas más frecuentes, seguidos de artralgias y dolor abdominal en las tres enfermedades.

Los signos clínicos más frecuentes en dengue y leptospirosis, fueron: prueba de torniquete positiva, adenopatías, melenas, epistaxis, hematemesis, esplenomegalia, oliguria y anuria. Asimismo, se evidenció mayor compromiso cardiopulmonar y confusión mental en la leptospirosis. 
Cuadro 2. Distribución de pacientes con síndrome febril según diagnóstico, en tres municipios del Urabá antioqueño, 2007-2008

\begin{tabular}{|c|c|c|c|c|c|c|}
\hline \multirow{3}{*}{ Variable } & \multicolumn{6}{|c|}{ Diagnóstico } \\
\hline & \multicolumn{2}{|c|}{ Apartadó } & \multicolumn{2}{|c|}{ Turbo } & \multicolumn{2}{|c|}{ Necoclí } \\
\hline & $\mathrm{n}=77$ & (\%) & $\mathrm{n}=80$ & (\%) & $n=43$ & (\%) \\
\hline Síndrome febril no determinado & 39 & $(50,6)$ & 37 & $(46,2)$ & 31 & $(72,1)$ \\
\hline Dengue & 29 & $(37,7)$ & 35 & $(43,7)$ & 11 & $(25,6)$ \\
\hline Leptospira & 13 & $(16,9)$ & 14 & $(17,5)$ & 0 & $(0,0)$ \\
\hline Rickettsia & 1 & $(1,3)$ & 4 & $(5,0)$ & 1 & $(2,3)$ \\
\hline \multicolumn{7}{|l|}{ Coinfecciones } \\
\hline Leptospira/dengue & 4 & $(5,2)$ & 8 & $(10,0)$ & 0 & $(0,0)$ \\
\hline Rickettsia/dengue & 0 & $(0,0)$ & 2 & $(2,5)$ & 0 & $(0,0)$ \\
\hline Leptospira/rickettsia/dengue & 1 & $(1,3)$ & 0 & $(0,0)$ & 0 & $(0,0)$ \\
\hline
\end{tabular}

Cuadro 3. Características sociodemográficas de los diagnósticos encontrados en tres municipios del Urabá antioqueño, 20072008.

\begin{tabular}{lrrrrrr}
\hline & \multicolumn{7}{c}{ Diagnósticos } \\
\cline { 2 - 7 } Variable & \multicolumn{2}{c}{ Dengue } & \multicolumn{2}{c}{ Leptospirosis } \\
n=119 & $(\%)$ & \multicolumn{2}{c}{ Rickettsiosis } \\
& $\mathbf{n}=1 \%)$ & $\mathbf{n = 1 1 9}$ & $(\%)$ \\
\hline Pacientes & 82 & $(68,9)$ & 31 & $(26,0)$ & 6 & $(5,0)$ \\
Sexo & & & & & & \\
$\quad$ Masculino & 55 & $(67,1)$ & 19 & $(61,3)$ & 5 & $(83,3)$ \\
Femenino & 27 & $(32,9)$ & 12 & $(38,7)$ & 1 & $(16,7)$ \\
Edad & & & & & & \\
$<10$ & 12 & $(14,6)$ & 5 & $(16,1)$ & 1 & $(16,7)$ \\
$11-15$ & 6 & $(7,3)$ & 3 & $(9,7)$ & 0 & $(0,0)$ \\
$16-20$ & 13 & $(15,8)$ & 6 & $(19,3)$ & 2 & $(33,3)$ \\
$21-25$ & 5 & $(6,1)$ & 3 & $(9,7)$ & 0 & $(0,0)$ \\
$26-30$ & 10 & $(12,2)$ & 3 & $(9,7)$ & 0 & $(0,0)$ \\
$30-40$ & 16 & $(19,5)$ & 7 & $(22,6)$ & 0 & $(0,0)$ \\
$>40$ & 20 & $(24,4)$ & 4 & $(12,9)$ & 3 & $(50,0)$ \\
Zona & & & & & & \\
$\quad$ Rural & 31 & $(37,8)$ & 9 & $(29,0)$ & 4 & $(66,7)$ \\
Urbana & 51 & $(62,2)$ & 22 & $(71,0)$ & 2 & $(33,3)$ \\
\hline
\end{tabular}

En el análisis multivariado se encontró asociación entre dengue y los siguientes factores: el mes febrero de 2008 (OR=6,56; $\quad \mathrm{IC}_{95 \%}=1,22$ $35,27 ; p<0,028)$, el sexo masculino (OR=2,41; $\left.\mathrm{IC}_{95 \%}=1,14-5,08 ; p<0,021\right)$ y la humedad relativa alta $(>80 \%)\left(\mathrm{OR}=8,98 ; \mathrm{IC}_{95 \%}=1,142-70,65 ; \mathrm{p}<0,037\right)$. Igualmente, para leptospirosis se estimó la fecha de consulta en febrero de 2008 (OR=6,0; IC ${ }_{95 \%}=1,50$ $23,86$; $p<0,011)$ como factor de riesgo y en los casos de rickettsiosis no se observó ningún factor asociado con la enfermedad.

\section{Discusión}

El presente estudio permitió demostrar por pruebas serológicas, la circulación de arenavirus. Estos agentes virales no habían sido registrados en la zona como causantes de síndrome febril, observándose seroconversión en un paciente de Turbo. Igualmente, se confirmó la presencia de rickettsias, virus del dengue y leptospiras como agentes causantes del síndrome febril no palúdico $y$, de hantavirus, previamente demostrada en roedores de la zona. En 53,2\% de los casos febriles con gota gruesa negativa para paludismo, no fue posible identificar ningún agente causal, hallazgo un poco más frecuente a lo reportado en estudios previos en Perú, donde no se determinó el agente en $34,6 \%$ y $34,2 \%$ de los pacientes febriles $(16,17)$. El amplio porcentaje de casos que permanece con causa desconocida en estas investigaciones, sugiere que se deberían ampliar los métodos de diagnóstico para otros agentes de presentación infrecuente o para otros trastornos que podrían ser causantes comunes de síndrome febril, tales como influenza, encefalitis, salmonelosis o hepatitis (18).

Se diagnosticó el virus del dengue como origen de la infección con más frecuencia que otros agentes, lo cual está de acuerdo con los reportes de incidencia de esta enfermedad, considerada como endemoepidémica en el país (19), con una tendencia a su aumento en los últimos 10 años, al pasar de 22.775 casos registrados en el año 2000 a más de 150.000 casos en el 2010. Esta situación pone de manifiesto que, en el país, el dengue sigue siendo una seria preocupación para la salud pública (4).

En relación con la frecuencia de infección con dengue y el sexo, los resultados del presente trabajo contrastan con un estudio de seroprevalencia llevado a cabo en los municipios del Urabá antioqueño en 1999 (8), donde se encontró una diferencia estadísticamente significativa con una mayor frecuencia de mujeres infectadas por dengue $(64,6 \%)$ con respecto a la de los hombres (51,8\%). Del mismo modo, en un estudio de los pacientes 
Cuadro 4. Características clínicas de la población estudiada en los establecimientos de salud en tres municipios del Urabá antioqueño, 2007-2008

\begin{tabular}{|c|c|c|c|c|c|}
\hline \multirow[b]{2}{*}{ Signos y síntomas } & \multicolumn{2}{|c|}{ Toda la población } & \multicolumn{3}{|c|}{ Según enfermedad } \\
\hline & $\mathbf{n}$ & $(\%)$ & $\begin{array}{l}\text { Dengue } \\
n=82 \\
(\%)\end{array}$ & $\begin{array}{l}\text { Leptospirosis } \\
\qquad \mathrm{n}=31 \\
(\%)\end{array}$ & $\begin{array}{c}\text { Rickettsiosis } \\
\begin{array}{c}\mathrm{n}=6 \\
(\%)\end{array}\end{array}$ \\
\hline Fiebre & 218 & 99,1 & 100,0 & 96,8 & 100,0 \\
\hline Cefalea & 211 & 95,9 & 89,8 & 96,8 & 100,0 \\
\hline Mialgias & 205 & 93,2 & 88,6 & 96,8 & 100,0 \\
\hline Artralgias & 205 & 93,2 & 89,8 & 96,8 & 100,0 \\
\hline Dolor abdominal & 161 & 73,2 & 71,6 & 71,0 & 66,7 \\
\hline Anorexia & 160 & 72,7 & 67,0 & 77,4 & 66,7 \\
\hline Dolor retroocular & 135 & 61,4 & 60,2 & 71,0 & 50,0 \\
\hline Náuseas/vómito & 128 & 58,2 & 59,1 & 58,1 & 83,3 \\
\hline Tos & 116 & 52,7 & 48,9 & 45,2 & 50,0 \\
\hline Congestión nasal & 101 & 45,9 & 36,4 & 45,2 & 33,3 \\
\hline Coluria & 86 & 39,1 & 43,2 & 38,7 & 50,0 \\
\hline Dolor de garganta & 82 & 37,3 & 29,5 & 32,3 & 33,3 \\
\hline Diarrea & 72 & 32,7 & 33,0 & 35,5 & 16,7 \\
\hline Ictericia & 70 & 31,8 & 34,1 & 32,3 & 33,3 \\
\hline Erupciones & 39 & 17,7 & 18,2 & 19,4 & 33,3 \\
\hline Prueba del torniquete positiva & 27 & 12,3 & 6,8 & 22,6 & 0,0 \\
\hline Adenopatía & 26 & 11,8 & 8,0 & 16,1 & 0,0 \\
\hline Petequias & 25 & 11,4 & 9,1 & 16,1 & 16,7 \\
\hline Complicaciones & 22 & 10,0 & 14,8 & 3,2 & 33,3 \\
\hline Melena & 13 & 5,9 & 5,7 & 6,5 & 0,0 \\
\hline Dificultad respiratoria & 13 & 5,9 & 5,7 & 3,2 & 16,7 \\
\hline Púrpura equimosis & 12 & 5,4 & 5,7 & 9,7 & 16,7 \\
\hline Hepatomegalia & 11 & 5,0 & 6,8 & 6,5 & 16,7 \\
\hline Epistaxis & 8 & 3,6 & 3,4 & 3,2 & 0,0 \\
\hline Hematuria & 8 & 3,6 & 4,5 & 6,5 & 16,7 \\
\hline Hematemesis & 4 & 1,8 & 2,3 & 3,2 & 0,0 \\
\hline Esplenomegalia & 4 & 1,8 & 3,4 & 6,5 & 0,0 \\
\hline Compromiso cardiopulmonar & 4 & 1,8 & 2,3 & 0,0 & 16,7 \\
\hline Oliguria & 3 & 1,4 & 2,3 & 6,5 & 0,0 \\
\hline Confusión mental & 3 & 1,4 & 1,1 & 0,0 & 16,7 \\
\hline Metrorragias & 1 & 0,4 & 0,0 & 0,0 & 0,0 \\
\hline Ascitis & 1 & 0,4 & 0,0 & 0,0 & 0,0 \\
\hline Anuria & 1 & 0,4 & 1,1 & 3,2 & 0,0 \\
\hline
\end{tabular}

hospitalizados por dengue en Apartadó, hubo una mayor frecuencia de casos del sexo femenino (55,6\%), comparado con los pacientes masculinos $(44,4 \%)(20)$. No obstante, la distribución de la enfermedad por sexo siempre ha mostrado una mayor incidencia en el sexo masculino que en el femenino, con una razón hombre:mujer que oscila entre 2,5 y $1,5: 1$ (21).

Con respecto a la leptospirosis, vale la pena mencionar que la identificación del serogrupo por la prueba de microaglutinación es esencial para el entendimiento de la epidemiología de la enfermedad, ya que proporciona información sobre el reservorio animal que puede estar involucrado en la transmisión del agente en una zona determinada. En este caso, fueron más frecuentes las reacciones positivas contra el serogrupo
Tarassovi (14), en estrecha relación con bovinos y porcinos. Con respecto a esto, se podría inferir que los mamíferos relacionados con el serogrupo mencionado anteriormente podrían estar jugando un papel en la epidemiología de esta enfermedad, en la región de Urabá.

Desde el punto de vista de las enfermedades concomitantes, vale resaltar que en $12,5 \%$ de los casos (15/120) hubo infecciones simultáneas, siendo más frecuente la coinfección por dengue y leptospirosis (12/15). Esta coinfección, frecuentemente reportada como concurrente en un mismo paciente, podría explicarse porque ambas enfermedades suelen ser endémicas en áreas tropicales y clínicamente indistinguibles en su presentación como síndrome febril agudo. En el estudio de pacientes hospitalizados con dengue 
en Apartadó, se documentaron enfermedades concomitantes en $40 \%(18 / 45)$ de los casos, siendo la combinación de dengue y malaria la más frecuente (20).

Aunque las principales manifestaciones clínicas de estos cuadros febriles agudos suelen ser similares y prevalecieron con mayor frecuencia los síntomas generales (fiebre, cefalea, mialgias, artralgias y dolor abdominal), sí vale la pena destacar la frecuencia de manifestaciones hemorrágicas espontáneas o provocadas por la prueba del torniquete, en los casos de dengue y leptospirosis. Estas manifestaciones hemorrágicas usualmente se han asociado con dengue, particularmente la prueba de torniquete positiva. En el presente estudio hubo una mayor frecuencia de resultados positivos de esta prueba en los casos de leptospirosis (22,6\%), en comparación con los de dengue (6,8\%). Valdría la pena retomar este procedimiento diagnóstico en forma rutinaria para la orientación del médico tratante hacia la presencia de manifestaciones hemorrágicas en estos cuadros febriles agudos con sospecha de leptospirosis.

El inicio de los signos clínicos en febrero de 2008, fue un factor de riesgo tanto para dengue como para leptospirosis, lo cual puede relacionarse con los factores climáticos que se presentaron en esa época, ya que hay pruebas de que la estacionalidad y el nivel de precipitación de un área determinada afectan la disponibilidad de sitios de multiplicación para mosquitos y otros vectores que tienen estados inmaduros en el agua; este es el caso particular del dengue (22). Asimismo, en el caso de la leptospirosis también se han observado brotes relacionados con la estacionalidad o la transición de verano a invierno (23).

Con respecto a la infección por Ricketsia spp., cuatro de los seis casos se presentaron en el municipio de Turbo, donde se reportó el último brote de la región de Urabá en 2008. A este respecto, llama la atención la presencia de cinco casos en hombres comparados con uno solo en mujeres, lo que coincidiría con una mayor exposición al vector en personas que trabajan en labores agrícolas. No obstante, la ausencia de casos positivos para Rickettsia spp. en el rango entre los 20 y los 40 años de edad, contrasta con la explicación previa y podría insinuar que el número de individuos simplemente es insuficiente para especular sobre la significancia estadística de las diferencias encontradas. Además, aún existen muchos vacíos en el conocimiento de las características eco-epidemiológicas de este agente en zonas posiblemente endémicas para su infección (huéspedes vulnerables, vectores y reservorios) y, por lo tanto, también se desconocen los factores de riesgo asociados con una mayor frecuencia de infección en la población expuesta.

Los hallazgos del presente estudio reafirman la importancia del virus del dengue, Leptospira spp, y Rickettsia spp., como agentes causantes del síndrome febril en la región del Urabá. La amplia superposición en las manifestaciones clínicas de estas enfermedades (cuadro 4), enfatiza la importancia de mejorar la disponibilidad y oportunidad de los recursos diagnósticos existentes. Estos resultados también reiteran la necesidad de desarrollar nuevas herramientas diagnósticas para dilucidar muchos de los casos que permanecen sin identificación, y que generan una importante cuota de enfermedad e incapacidad sin valoración económica y social.

Se requiere, además, de la caracterización clínica de entidades emergentes y reemergentes aún poco investigadas, así como de exámenes de laboratorio que ayuden a establecer las condiciones de mayor riesgo. Valdría la pena, igualmente, sensibilizar al personal de salud respecto a la presencia y el posible incremento de infecciones zoonóticas "olvidadas" o "descuidadas", la determinación de los síntomas que ayuden a detectar su presencia y valorar su pronóstico, así como sobre el establecimiento de su tratamiento oportuno, en particular, para casos de fácil aproximación terapéutica, tales como los de leptospirosis y rickettsiosis.

\section{Conflicto de intereses}

Los autores del presente manuscrito declaran que no existe conflicto de intereses para la publicación de los resultados de este estudio.

\section{Financiación}

Este trabajo fue patrocinado por medio de la estrategia de sostenibilidad 2009-2010 del CODI de la Universidad de Antioquia, por el ICMTCES (Instituto Colombiano de Medicina TropicalCES), por la Fundación para la Promoción de la Investigación y la Tecnología del Banco de la República (proyecto 2387) y por Colciencias (proyecto 111534319203).

\section{Referencias}

1. World Health Organization. Epidemiological surveillance and international health regulations-1998. Fecha de consulta: 25 de marzo de 2012. Disponible en: http://www. searo.who.int/LinkFiles/Publication_126-EpidemiologicalSurveillance-IHR.pdf 
2. Instituto Nacional de Salud. Manual de procedimientos enfoque sindrómico para el diagnóstico de laboratorio durante brotes- 2005. Fecha de consulta: 25 de marzo de 2012. Disponible en: http://www.bvs.ins.gob.pe/insprint/ salud_publica/nor_tec/42.pdf

3. Pacheco A, Quiñones ML, Serrato IM, Rivas FA. Evaluación preliminar de la Estrategia de información, comunicación y educación para el control del Aedes aegypti, en La Dorada, Colombia. Rev Salud Pública. 2010;12:38090. http://dx.doi.org/10.1590/S0124-00642010000300004

4. Velandia ML, Castellanos JE. Virus del dengue: estructura y ciclo viral. Infectio. 2011;15:33-43.

5. Agudelo-Flórez P, Restrepo BN, Arboleda M. Situación de la leptospirosis en el Urabá antioqueño colombiano: estudio seroepidemiológico y factores de riesgo en población general urbana. Cad Saúde Pública. 2007;23:2094-102. http://dx.doi.org/10.1590/S0102-311X2007000900017

6. Agudelo-Flórez P. Leptospirosis humana en Colombia: la experiencia del Instituto Colombiano de Medicina Tropical CES. Rev CES Med. 2007;21:55-5.

7. Padmanabha $\mathbf{H}$, Hidalgo $\mathbf{M}$, Valbuena $\mathbf{G}$, Castañeda E, Galeano A, Puerta H, et al. Geographic variation in risk factors for SFG rickettsial and leptospiral exposure in Colombia. Vector Borne Zoonotic Dis. 2009;9:483-90. http:// dx.doi.org/10.1089/vbz.2008.0092

8. Restrepo BN, Arboleda M, Lopera T. Estudio seroepidemiológico de dengue en la región del Urabá antioqueño, Colombia. Infectio. 2004;8:255-62.

9. Dirección Seccional de Salud de Antioquia. Eventos de salud pública. 2010. Fecha de consulta: 25 de marzo de 2012. Disponible en: http://www.dssa.gov.co/index.php/ estadisticas/eventos-de-salud-publica

10. Dirección Seccional de Salud de Antioquia. Circulares 2006. Fecha de consulta: 25 de marzo de 2012. Disponible en: http://www.dssa.gov.co/index.php/legislacion/circulares

11. Suárez R, Hidalgo M, Niño N, González C, Vesga JF, Orejuela L, et al. Las rickettsias como agentes etiológicos de entidades febriles no diagnosticadas en Colombia. $1^{\text {a }}$ edición. Bogotá: Ediciones Uniandes; 2008. p. 1-94.

12. Londoño AF, Díaz FJ, Agudelo P, Levis S, Rodas JD. Genetic evidence of hantavirus infections in wild rodents from northwestern Colombia. Vector Borne Zoonotic Dis. 2011;11:701-8. http://dx.doi.org/10.1089/vbz.2010.0129

13. Agudelo P, Restrepo M, Lotero MA. Evaluación de la prueba de inmunofluorescencia indirecta para el diagnóstico de leptospirosis humana. Biomédica. 2006;26:216-23.
14. World Health Organization. International Leptospirosis Society. Human leptospirosis: Guidance for diagnosis, surveillance and control - 2003. Fecha de consulta: 25 de marzo de 2012. Disponible en: http://www.med.monash. edu.au/microbiology/staff/adler/ilspage.html

15. Horta MC, Labruna MB, Sangioni LA, Vianna MC, Gennari SM, Galvão MA, et al. Prevalence of antibodies to spotted fever group Rickettsiae in humans and domestic animals in a Brazilian spotted fever-endemic area in the state of Sao Paulo, Brazil: serologic evidence for infection by Rickettsia rickettsii and another spotted fever group Rickettsia. Am J Trop Med Hyg. 2004;71:93-7.

16. Ministerio de Salud del Perú, Instituto de Investigación de Enfermedades Tropicales de la Marina de los Estados Unidos, Universidad Nacional Mayor de San Marcos, Universidad Peruana Cayetano Heredia. Perfil etiológico del síndrome febril en áreas de alto riesgo de transmisión de enfermedades infecciosas de impacto en salud pública en el Perú, 2000-2001. Rev Peru Med Exp Salud Pública. 2005;22:165-74.

17. Troyes L, Fuentes L, Troyes M, Canelo L, García M, Anaya $\mathrm{E}$, et al. Etiología del síndrome febril agudo en la provincia de Jaén, Perú 2004- 2005. Rev Peru Med Exp Salud Pública. 2006;23:5-11.

18. Ministerio de Salud. Estratos ecológicos, estratos epidemiológicos y análisis de situación de salud. El caso de Perú - 2005. Fecha de consulta: 25 de marzo de 2012. Disponible en: http://www.bvs.ins.gob.pe/print/carrion/ ppt/31.pdf

19. Díaz FA, Martínez RA, Villar LA. Criterios clínicos para diagnosticar el dengue en los primeros días de enfermedad. Biomédica. 2006;26 22-30.

20. Arboleda M, Campuzano M, Restrepo BN, Cartagena G. Caracterización clínica de los casos de dengue hospitalizados en la Empresa Social del Estado Hospital "Antonio Roldán Betancur", Apartadó, Antioquia, 2000. Biomédica. 2006;26:286-94.

21. Guha-Sapir D, Schimmer B. Dengue fever: New paradigms for a changing epidemiology. Emerg Themes Epidemiol. 2005;2:1-10. http://dx.doi.org/10.1186/1742-7622-2-1

22. Gage KL, Burkot TR, Eisen RJ, Hayes EB. Climate and vector borne diseases. Am J Prev Med. 2008;35:436-50. http://dx.doi.org/10.1016/j.amepre.2008.08.030.

23. Levett PN. Leptospirosis. Clin Microbiol Rev. 2001;14:296326. 